\title{
A THEOREM IN FINITE PROJECTIVE GEOMETRY AND AN APPLICATION TO STATISTICS ${ }^{1}$
}

\section{ESTHER SEIDEN}

R. C. Bose showed that certain statistical problems of design of experiments can be attacked fruitfully by interpreting the statistical terms involved in terms of finite geometries. In particular, the geometrical interpretation proved useful when applied to the problem of determining the maximum number of factors which can be accommodated in symmetrical factorial design without confounding the degrees of freedom belonging to interactions of a given order or lesser.

In a paper Mathematical theory of factorial design (Sankhya vol. 8 (1947) pp. 107-166) R. C. Bose proved that the maximum number, $m_{t}(r, s)$, of factors which can be accommodated in symmetrical factorial design in which each factor is at $s=p^{n}$ levels ( $p$ being a positive prime and $n$ a positive integer) and each block is of size $s^{r}$, without confounding any degrees of freedom belonging to any interaction involving $t$ or lesser number of factors, is given by the maximum number of points of a finite projective space $P G(r-1, s)$ (of $r$ homogenous co-ordinates each of which is capable of $s$ values) such that no $t$ of the chosen points are conjoint (lie in a space of $t-2$ dimensions). Furthermore, R. C. Bose proved in the above mentioned paper that $m_{3}(4, s)=s^{2}+1$ when $s$ is a power of an odd prime and $s^{2}+1$ $\leqq m_{3}(4, s) \leqq s^{2}+s+2$ when $s=2^{n}, n>1$. This inequality gives, for $s=4,17 \leqq m_{3}(4,4) \leqq 22$. It is the purpose of this paper to prove that $m_{3}(4,4)=17$. In other words: the maximum number of points of $P G(3,4)$ such that no 3 of them are collinear cannot exceed 17 (Theorem 5).

In order to make the reading of this paper independent of the literature on finite geometry we explain shortly the $P G(3,4)$. The points in $P G(3,4)$ can be represented by attaching to it a Galois field with 4 elements, $G F(4)$, built with the help of an irreducible polynomial, $\epsilon^{2}+\epsilon+1=0$, where a point is understood to be an ordered quadruple of elements of $G F(4)$, not all zero. Two points $\left(x_{1}, x_{2}, x_{3}, x_{4}\right)$ and $\left(y_{1}, y_{2}, y_{3}, y_{4}\right)$ are defined to be identical if there exists a nonzero element of $G F(4)$, say $\rho$, such that $x_{i}=\rho y_{i}, i=1,2,3,4$. Thus, the total number of points is seen to be $\left(4^{4}-1\right) / 3=85$. By a line passing through two different points $\left(x_{1}, x_{2}, x_{3}, x_{4}\right)$ and $\left(y_{1}, y_{2}, y_{3}, y_{4}\right)$

Received by the editors January 11, 1949.

1 This work was supported in part by the Office of Naval Research. 
is understood the set of points $\left(\mu x_{1}+\lambda y_{1}, \mu x_{2}+\lambda y_{2}, \mu x_{3}+\lambda y_{3}, \mu x_{4}+\lambda y_{4}\right)$ where $\mu, \lambda$ are elements of $G F(4)$. It is seen that the number of points on a line is 5. Defining, similarly, what is meant by a plane, the number of planes passing through a line will be 5 and the number of points on a plane 21. Finally, the remark that the observations usual in projective geometry concerning the choice of the coordinate system hold true in $P G(3,4)$.

The following two theorems are known to hold in $P G(3,4)$.

Theorem 1. The three diagonal points of a nondegenerate plane quadrangle are distinct and lie on a line, say $L$.

The proof follows by taking coordinates such that the points of the quadrangle are $(0,1,0,0),(0,0,1,0),(0,0,0,1),(0,1,1,1)$. In this case the diagonal points are $(0,1,1,0),(0,1,0,1),(0,0,1,1)$ and the equation of $L$ becomes evidently $x_{1}=0, x_{2}+x_{3}+x_{4}=0$.

Theorem 2. In every plane there are 6 "no three collinear" points.

$$
(0,1,0,0),(0,0,1,0),(0,0,0,1),(0,1,1,1),\left(0,1, \epsilon, \epsilon^{2}\right),\left(0,1, \epsilon^{2}, \epsilon\right)
$$
are six such points of the plane $x_{1}=0$. Evidently the last two points lie on $L$ and differ from the above-mentioned three diagonal points. Moreover, together with them they exhaust the points of $L$.

TheOREM 3. Any 4 no three collinear points $Q_{1}, Q_{2}, Q_{3}, Q_{4}$ in a plane determine uniquely 2 more points, say $Q_{6}, Q_{6}$, such that $Q_{1}, Q_{2}, \cdots, Q_{6}$ are no three collinear points.

Let us consider the five lines in the plane passing through $Q_{1}$. They exhibit all the points of the plane. Three of them coincide with the lines determined by the pairs of points $Q_{1} Q_{2}, Q_{1} Q_{3}, Q_{1} Q_{4}$. Each of the remaining two lines meets the lines $Q_{2} Q_{3}, Q_{2} Q_{4}, Q_{3} Q_{4}$ in three distinct points. Thus on each of these two lines there remains just one point, say $Q_{6}, Q_{6}$, respectively which form together with $Q_{1}, \cdots, Q_{4}$ a set of no three collinear points.

Definitron. Any set of 6 no three collinear points of a plane will be called 6-basic.

Theorem 4. Consider two planes $p_{1}$ and $p_{2}$ intersecting in a line determined by two points $U_{1}, U_{2}$ and 4 more points $Q_{1}, Q_{2}, Q_{3}, Q_{4}$ of $p_{1}$ and $R_{1}, R_{2}, R_{3}, R_{4}$ of $p_{2}$ so that $U_{1}, U_{2}, Q_{1}, Q_{2}, Q_{3}, Q_{4}$ as well as $U_{1}, U_{2}, R_{1}$, $R_{2}, R_{3}, R_{4}$ are 6-basic. Then,

1. The 16 lines $Q_{i} R_{j}(i, j=1,2,3,4)$ are distinct.

2. They can be distributed into four sets of 4 lines so that the 4 lines of each set intersect in exactly one point. 
3. If $Q_{i} Q_{j}$ and $R_{l} R_{m}$ are coplanar, then they meet the line $U_{1} U_{2}$ in the same point.

4. The four points of intersection of the lines $Q_{i} R_{j}$, say $O_{1}, O_{2}, O_{3}, O_{4}$, lie on a plane, say $p_{3}$, passing through $U_{1} U_{2}$.

5. $U_{1}, U_{2}, O_{1}, O_{2}, O_{3}, O_{4}$ are 6-basic and the role of the sets of $Q$ 's or $R$ 's or $O$ 's can be interchanged.

Proof. 1 . If a line $\lambda$ given by $Q_{i} R_{j}$ were identical with that given by $Q_{k} R_{l}$, then, assuming without loss of generality $i \neq k$, it would follow that points $Q_{i}, Q_{k}, R_{j}$ lie on $\lambda$ and consequently that $R_{j}$ is in $p_{1}$ against the assumption.

2. There exists a two-to-two correspondence between the 6 lines $Q_{i} Q_{j}$ and the 6 lines $R_{k} R_{l}$ if we associate with line $Q_{i} Q_{j}$ any of the two lines $R_{k} R_{l}$ which pass through the same one of the three points of $L$ other than $U_{1}, U_{2}$. By this correspondence the points of a given triangle $Q_{i} Q_{j} Q_{s}$ are made to correspond to those of an arbitrary triangle $R_{k} R_{l} R_{m}$ in a unique way. For $Q_{i} Q_{j}$ corresponds to at most one side, say $R_{k} R_{l} ; Q_{i} Q_{s}$ corresponds to another side, say $R_{k} R_{m}$; hence the intersection $Q_{i}$ of $Q_{i} Q_{j}$ and $Q_{i} Q_{z}$ corresponds to the intersection of $R_{k} R_{l}$ and $R_{k} R_{m}$ which is $R_{k}$; and accordingly $Q_{j}$ must correspond to $R_{l}, Q_{\text {. }}$ to $R_{m}$; it is seen that also line $Q_{j} Q_{s}$ corresponds to line $R_{l} R_{m}$ because these lines must pass through the remaining one of the three points of $L$ other than $U_{1}, U_{2}$. Now we apply the converse of Desargue's theorem to the triangles $Q_{i} Q_{j} Q_{s}$ and $R_{k} R_{l} R_{m}$ and obtain that the lines $Q_{i} R_{k}, Q_{j} R_{l}, Q_{s} R_{m}$ pass through one point, say $O$; furthermore, if $Q^{\prime}$ is the fourth point of the $Q$ set, $R^{\prime}$ the fourth point of the $R$ set, then $Q^{\prime} R^{\prime}$ passes also through $O$; for the triangle $Q_{i} Q_{j} Q^{\prime}$ corresponds to $R_{k} R_{l} R^{\prime}$ necessarily point by point in the same order since line $Q_{i} Q^{\prime}$ is different from $Q_{i} Q_{j}$ and $Q_{i} Q_{s}$, hence these three lines intersect with $L$ at three distinct points other than $U_{1}, U_{2}$, thus determining the intersection of $L$ with $Q_{i} Q^{\prime}$ by exclusion, and the same holds for $R_{k} R^{\prime}$. Applying the converse of Desargue's theorem to the triangles $Q_{i} Q_{j} Q^{\prime}$ and $R_{k} R_{l} R^{\prime}$, we find that $Q_{i} R_{k}, Q_{j} Q_{l}, Q^{\prime} R^{\prime}$ pass through a point which necessarily is $O$. Making any of the four triangles formed by 3 out of the four points $R_{1}, R_{2}, R_{3}, R_{4}$ correspond to the triangle $Q_{1}, Q_{2}, Q_{3}$ in the indicated manner, we obtain 4 points $O_{1}, O_{2}, O_{3}, O_{4}$ as points of perspectivity. They are distinct, for if, for example, the lines $Q_{1} R_{1}$, $Q_{2} R_{2}, Q_{3} R_{3}, Q_{4} R_{4}$ meet at $O_{1}$, then a line $Q_{i} R_{j}$, where $i \neq j$, cannot pass through $O_{1}$ because then $R_{i}, R_{j}, Q_{i}$ would be collinear and consequently $Q_{i}$ would lie on the plane $p_{2}$ against the assumption.

3. Two coplanar lines $Q_{i} Q_{j}$ and $R_{l} R_{m}$ meet the line $L$ (intersection of the planes $p_{1}$ and $p_{2}$ ) in the same point, because otherwise the planes $p_{1}$ and $p_{2}$ would be identical against the assumption. 
4. Given any two points of the set $O_{1}, O_{2}, O_{3}, O_{4}$, say $O_{1}$ and $O_{2}$, there exist two points $Q_{i}, Q_{j}$ and two points $R_{l}, R_{m}$ such that the line $Q_{i} R_{l}$ meets $Q_{j} R_{m}$ in $O_{1}$ and $Q_{i} R_{m}$ meets $Q_{j} R_{l}$ in $O_{2}$. Take $Q_{i}$ arbitrary, determine $R_{l}$ so that the line $Q_{i} R_{l}$ passes through $O_{1}$; determine $R_{m}$ so that $Q_{i} R_{m}$ passes through $\mathrm{O}_{2}$; then $\mathrm{O}_{2} R_{l}$ must pass through another $Q$, say $Q_{j} . Q_{i}, Q_{j}, R_{l}, R_{m}$ form evidently a nondegenerate plane quadrangle and thus the line $Q_{i} Q_{j}$ meets the line $R_{l} R_{m}$ in a point $P$ and, by Theorem $1, O_{1}, O_{2}, P$ are collinear. But $P$ lies, by 3 , on the line $L$, thus $O_{1}, O_{2}, U_{1}, U_{2}$ are coplanar. The same holds for any $O_{i}, O_{j}, U_{1}, U_{2}$, showing that all $O_{1}, O_{2}, O_{3}, O_{4}$ lie in a plane through $U_{1}, U_{2}$, say $p_{3}$.

5. Line $U_{1} U_{2}$ does not contain an $O$; furthermore any line $O_{i} O_{j}$ meets the line $U_{1} U_{2}$ at a point $P$ different from $U_{1}$ and $U_{2}$, so that no $U$ lies on a line joining two $O$ 's and no $O$ lies on a line joining two $U$ 's. It remains to show that no three of the $O$ 's are collinear. Assume without loss of generality that

\section{$O_{1}$ is intersection of $Q_{1} R_{1}$ with $Q_{2} R_{2}$, \\ $\mathrm{O}_{2}$ is intersection of $Q_{1} R_{2}$ with $Q_{2} R_{1}$, \\ $O_{3}$ is intersection of $Q_{1} R_{3}$ with $Q_{2} R_{4}$.}

Now if $O_{1}, O_{2}, O_{3}$ were collinear, then $R_{1}, R_{2}, R_{3}$ would be obtained as intersections of their plane (which does not contain $Q_{1}$ ) with the plane $Q_{1} O_{1} O_{2} O_{3}$; hence $R_{1}, R_{2}, R_{3}$ would be collinear against assumption. Thus no three $O$ 's are collinear. It is seen that, under the assumption made above, $Q_{1}$ is the intersection of the lines $O_{1} R_{1}, O_{2} R_{2}, O_{3} R_{3}, O_{4} R_{4}$ and $R_{1}$ is the intersection of $O_{1} Q_{1}, O_{2} Q_{2}, O_{3} Q_{3}, O_{4} Q_{4}$. Evidently similar results follow for the remaining 3 groups of 4 lines $O_{i} R_{j}$ or $O_{i} Q_{j}$ respectively. Thus the rôles of the $O$ 's, $Q$ 's, and $R$ 's can be interchanged.

THEOREM 5. The maximum number of points which can be chosen in $P G(3,4)$ such that no three of the chosen points are collinear does not exceed 17.

Proof. Let $C$ be the set consisting of the maximum number of points no three collinear in $P G(3,4)$. Assume without loss of generality that $U_{1}, U_{2}$ belong to $C$. The proof is trivial in case when, among the 5 planes passing through $U_{1} U_{2}$, there do not exist 2 planes such that one of them contains 4 points, other than $U_{1}, U_{2}$, belonging to $C$ and the other plane at least 3 such points, because in this case the total number of points belonging to $C$ would readily be seen to be at most 17. Hence assume without loss of generality that $Q_{1}, Q_{2}, Q_{3}, Q_{4}$ as well as $R_{1}, R_{2}, R_{3}$ belong to $C$. Consider two cases (1) $R_{4}$ belongs 
to $C$, (2) $R_{4}$ does not belong to $C$.

In case 1 the 16 distinct lines $R_{i} Q_{j}(i, j=1,2,3,4)$ contain 16 points of each of the two planes, say $p_{4}, p_{5}$, other than $p_{1}, p_{2}, p_{2}$. Since the remaining 5 points of these planes belong to the line $L$, no points of these planes other than $U_{1}, U_{2}$ belong to $C$. $C$ can contain at most 4 additional points of $p_{3}$; accordingly, the total number of points in $C$ amounts to $14(2+3 \times 4=14)$.

In case 2 , the points of $p_{4}$ and $p_{5}$ which belong to $C$ are on the lines $Q_{i} R_{4}(i=1,2,3,4)$. Since the $Q$ 's belong to $C$ by assumption, there are at most 4 more points of these lines belonging to $C$. In addition at most 4 points other than $U_{1}, U_{2}$ of $p_{3}$ belong to $C$. Thus in this case the total number of points belonging to $C$ is at most $17(2+3+3$ $\times 4=17)$.

REMARK. It can be proved that in case 2 the total number of points belonging to $C$ is 13 .

Theorem 5 together with the inequality $m_{3}(4,4) \geqq 17$ proved by $R$. C. Bose gives the required equality, namely $m_{3}(4,4)=17$.

It is a pleasure to record the author's indebtedness to Professor $R$. C. Bose for suggesting this problem and to Professor Hans Lewy for frequent stimulating discussions which helped to prepare this paper.

It was proved after the paper was submitted to the editors that every set of 17 no three collinear points in $P G(3,4)$ forms a quadric surface.

University of California 